\section{Electric Impedance of Suspensions of Leucocytes}

Concentrated suspensions of rabbit white cells were prepared by the method described by Mudd, Lucké, McCutcheon and Strumia ${ }^{1}$. When obtained by this method, about 95 per cent of the cells are polymorphs. Microscopic examination and measurement of respiration showed that the cells were in a normal condition. The impedance measurements were made at $21 \cdot 4^{\circ} \mathrm{C}$. Suspensions having volume concentrations from 10 to 42 per cent, in buffered 0.95 per cent sodium chIoride, were used and the resistivity $R$ (ohm $-\mathrm{cm}$.) and parallel capacity $C$ (m $\mu \mathrm{F} / \mathrm{cm}$.) were determined from 0.25 to 16,000 kilocycles per sec. ${ }^{2,3}$ The values of $R$ and $C$, as functions of the frequency, are shown for a 42 per cent suspension in Fig. 1.

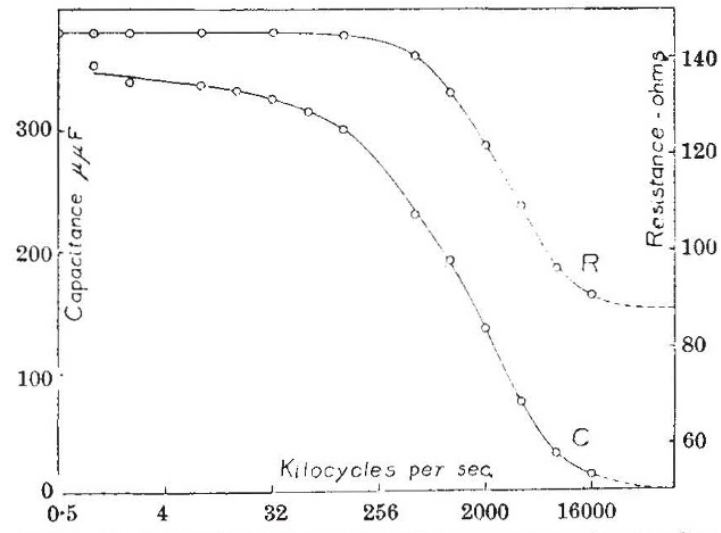

V1G. 1. Resistivity $(R)$ and capacity $(C)$ for a 42 per cent suspension of rabbit white cells (average diameter $10.5 \mu$ ) in 0.95 per cent sodium chloride. Resistivity of intercellular fluid $69 \cdot 0$ ohms.

The theoretical method of treating these observations has already been described ${ }^{2},^{3}$. We distinguish between that part of the impedance of the white cell which is attributable to the surface and that part uttributable to the interior. At low frequencies only the former part is effective and from the (approximate) constancy of $C$ and $R$, at these frequencies, it is eoncluded that the impedance at the surface is due to the presence of a poorly conducting membrane which acts as a static condenser. By the same criteria, the presence of such a membrane at the surface of the erythrocyte and of the yeast cell has previously been established ${ }^{2,3,4}$. The capacity per sq. $\mathrm{cm}$. of surface of the white cell can be calculated to be $1 \cdot 0 \pm 0 \cdot 1 \mu \mathrm{F} / \mathrm{cm}^{2}$. This value is the same as found for the surface membrane of the erythrocyte ${ }^{3}$, within the limits of experimental error. With the rather arbitrary value of 3 for the dielectric constant of the membrane, this capacity corresponds to a thickness of $27 \times 10^{-8} \mathrm{~cm}$.

From experience with other cells ${ }^{3,4}$, it is likely that $C$ and $R$ increase slightly at the lowest frequencies. The theoretical significance of this increase as related to the ionic permeability of the membrane has been diseussed ${ }^{3,4}$. Unfortunately, due to the rapid settling of the white cells, this increase cannot be accurately measured here, but it cannot be greatly different from that found for the red corpuscle. The decrease in $C$ and $R$ at about 128 kilocycles marks the point at which the interior of the cell makes its influence felt. The theoretical treatment is not sufficiently developed to allow us to recognise definitely any effects due to a polarisability of the membranes of the internal structures (nucleus, granules, etc.) but, within the present theoretical limitations, the impedance of the interior may be represented as a pure resistance, the specific value of which is calculated ${ }^{2}$ to be $140 \pm 10 \mathrm{ohm}-\mathrm{cm}$. $\left(21 \cdot 4^{\circ} \mathrm{C}\right.$.). This value is, within experimental error, the same as found for the mammalian red corpuscle ${ }^{5}$. Since the organic matter content is nearly the same for these two types of cell, this agreement further strengthons the view that the impedance of the membrane of the internal structures in the white cell is negligible at the high frequencies for which the impedance of the internal fluid can accurately be determined.

Hugo Fricke.

Howard J. Curtis.

Walter B. James Laboratory for Biophysies, Biological Laboratory, Cold Spring Harbor, New York.

${ }^{1}$ S. Mudd, B. Lucké, M. McCutcheon and M. Strumia, J. Exp. Med., 49, $779 ; 1929$.

${ }_{2}^{2}$ H. Fricke, 'Cold Spring Harbor Symposia on Quantitative Biology, 1, $117 ; 1933$.

${ }^{3}$ H. Fricke and H. J. Curtis, J. Gen. Physiol. (in press).

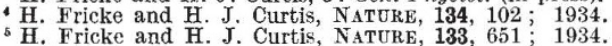

\section{Moving Striations}

WE have recently completed an investigation of the spectra of moving striations in the positive column of some glow discharges. Briefly, the method consists in forming an image of the positive column along the slit of a spectrograph, and covering and uncovering the latter by a mechanical shutter made synchronous with the moving striations by a photoelectric device. This arrangement gives spectra, the individual lines of which show a longitudinal variation of intensity corresponding to the bright and dark sections of the striations.

In the case of a discharge in pure argon, we have examined the dark period carefully for high series lines or series continua, and find them absent. It would therefore appear that recombination of ions and electrons in the gas phase is not important, and since it is known from work by Kenty ${ }^{1}$ on argon that such recombination is only appreciable when the average electron energies fall below about 0.5 e.v., we conclude that the electron energies in the dark periods exceed this amount. This is confirmed by the measurements of $\mathrm{Pupp}^{2}$ who, in a recent paper, has described the results of probe analysis of somewhat similar discharges and finds in the dark phase electron energies in excess of 1 e.v. Our optical results are also in accord with his electrical data in that we have found that when a trace of mercury is present, the mercury are lines 5461 A. and $4358 \mathrm{~A}$. (excitation potential $7 \cdot 6$ volts) persist strongly through the dark phase although argon lines (excitation potential greater than 12 volts) are, at best, extremely feeble there.

It is satisfactory to find that the optical and electrical studies of these still puzzling phenomena are mutually confirmatory to this extent.

R. H. SLOANE.

C. M. Minnis.

Department of Physics, Queen's University,

Belfast.

Feb. 2.

${ }^{1}$ Kenty, Phys. Rev., 32, 624; 1928.

2 Pupp, Phys. Z., 36, 61; 1935. 